\title{
E. C. G. Sudarshan at Texas
}

\author{
Austin Gleeson* \\ Department of Physics, The University of Texas at Austin, 2515 Speedway, Stop C1600 Austin, TX78712-1192, USA
}

\section{Before Texas}

Although George Sudarshan spent most of his scientific career at The University of Texas at Austin (UT Austin, USA), he was already an established and successful scientist with several enviable achievements to his credit before coming to Texas. In addition, what was it about Texas that would lure him from a good situation in Syracuse?

\section{George before Texas}

It started with his completing undergraduate studies at CMS College in Kottayam, Kerala and the affiliated Madras Christian College (MCC), in Tambaram in Tamil $\mathrm{Nadu}$, India, with a Bachelor of Arts with Honors. MCC is considered to be one of the most prestigious colleges in India. He, then, attended the University of Madras for his graduate studies taking only one year to earn his Master of Arts degree. In 1952, he moved to the Tata Institute of Fundamental Research (TIFR) in Mumbai to continue his graduate studies serving as a Research Assistant in a group studying the applications of the use of photographic techniques for the analysis of nuclear and elementary particle phenomena. Working with S. Biswas and B. Peters, he developed the variable cell length constant sagitta method for the determination of masses of charged particles undergoing multiple scattering in nuclear emulsions. He also developed the use of high-order differences to deal with distorted emulsions ${ }^{1}$.

The name he used at the time was his given name of Ennackal Chandy George or E. C. George. In scientific works published in the years following this period and his conversion to Hinduism, he used E. C. George Sudarshan where Sudarshan was chosen by him and is best translated as 'good looking'.

Robert E. Marshak, a leading theorist at Rochester University, USA, was travelling in India and visited TIFR and discovered Sudarshan. He was very impressed with Sudarshan and lured him to Rochester to complete a $\mathrm{Ph}$ D.

Arriving at Rochester in 1955, Sudarshan was immediately immersed into the excitement of fundamental theoretical physics at the highest level while working among world-leading physicists. This was also a time

*e-mail: gleeson@physics.utexas.edu when the experimentalists worldwide were discovering a wealth of newly identified particles. He was quickly engaged in the give-and-take of discovery in particle physics. It was quickly evident that there had to be some organizing principle to the pattern of the newly observed particles. He was among the first to realize that understanding patterns of the higher internal symmetries and their breaking was the key. With Okubo, Marshak and Weinberg, he made the first application of broken symmetries in particle physics by analysing the isotopic spin structure of the electromagnetic masses and moments ${ }^{2}$. He followed these with work on unitary symmetry.

For his graduate research project, Marshak assigned to him the task of untangling the very mixed situation of the understanding of the weak interactions. The four-fermion interaction model of weak decay articulated by Fermi offered many possibilities, too many and contradictory. By a comprehensive and detailed analysis of all the weak decay data, Sudarshan came to the far-reaching conclusion that a V-A structure was consistent with all but four of the experiments. This interaction was also the first time a chiral interaction favouring left-handed particles was articulated and parity, space inversion symmetry, was violated. Within a year, the four inconsistent experiments were repeated and found to be in agreement with Sudarshan's predictions.

The V - A parity violating form for the structure of the weak interactions was the first thread in the effort to unify the weak and electromagnetic interactions in what is now called the Standard Model. This was the extension by Marshak and Sudarshan, as well as Feynman and GellMann of the four-fermion model of Enrico Fermi's theory of weak interactions. The saga of priority is best summarized by Feynman:

'So I would like to say where we stand in our theories of weak interactions. We have a conventional theory of weak interactions invented by Marshak and Sudarshan, published by Feynman and Gell-Mann, and completed by Cabibbo ... ${ }^{3}$.

This work on $\mathrm{V}-\mathrm{A}$ has to be considered the most impressive $\mathrm{Ph} \mathrm{D}$ dissertation ever (note 1).

With the completion of his $\mathrm{PhD}$ at Rochester in two years, and the publication and extension of the $\mathrm{V}-\mathrm{A}$ theory of weak interactions, he took up residency as a Corporate Fellow at Harvard University from 1957 to 1959. During that period, he extended his work on weak interactions, parity violation and chiral invariance ${ }^{4}$ and 
began his work on inconsistency with the quantization of high spin fields ${ }^{5}$ and development of what is now called the Deser-Gilbert-Sudarshan representation of the vertex functions and other field theoretic amplitudes ${ }^{6}$.

In 1959, Sudarshan returned to Rochester as a faculty member. He immersed himself in the foundations of physics, in particular, the development of a formalism for classical dynamics to facilitate the understanding of quantum mechanical formulations of dynamics and the development of a relativistic quantum field theory. This work on classical dynamics was later articulated in his book with N. Mukunda ${ }^{7}$, written after he had moved to Texas. During these studies, he developed, with D. Currie and T. Jordan, the well-known 'no-go' theorems for interacting Hamiltonian particle theories in which he showed, by means of the Lie algebra formulation of classical dynamics, that Hamiltonian theories could not be manifestly Lorentz invariant and covariant and have interactions ${ }^{8}$.

At Rochester, he expanded his interests to include quantum optics and developed the 'optical equivalence theorem'. This result provides an experimentally transparent unification of the classical and quantum descriptions of electromagnetic phenomena, especially those dealing with lasers operating in the region of visible and nearvisible light ${ }^{9}$. This work is summarized in his book with J. Klauder ${ }^{10}$.

Sudarshan spent the period 1963-1964 as a Guest Professor at the University of Bern, Switzerland and at Brandeis University, USA. During that time, his interests expanded to cover several new and important areas of physics research. Most important has been the work on quantum theories with indefinite metric as a tool in constructing finite quantum field theories and their physical interpretation $^{11}$.

In 1964, he joined the faculty of Syracuse University, USA as Professor and Director of the Research Program in Elementary Particles. It was a time when the Department of Physics at Syracuse was growing, a new building was in the planning stages, and that was his opportunity to assemble a group of active physicists with related research interests. There were about ten people, one other faculty appointment, A. P. Balachandran, and four postdocs and five senior graduate students. Research funding was from the Atomic Energy Commission (AEC) (note 2 ). Research initiated in Rochester continued. Interests in quantum optics and symmetries were extended and, with Balachandran, Sudarshan extended his study of the Poincaré and the Galilei groups and the physical interpretation of their unphysical representations; this led to his interest in tachyons. With O. M. P. Bilaniuk and V. K. Deshpande, he reworked his original multiply rejected paper ${ }^{12}$. This paper caused quite a stir among the physics community.

He published a book with Marshak on elementary particle physics ${ }^{13}$.
The infinities of standard quantum field theory present interesting interpretation problems. This led to Sudarshan's interests in finite quantum electrodynamics which utilized an indefinite metric and particles of finite mass ${ }^{14}$. The origins of the use of non-invariance groups and dynamical symmetries were initiated ${ }^{15}$. He extended his work on modern optics ${ }^{16}$. With Mukunda and J. G. Kuriyan, he invented the master analytic representation method for constructing all the unitary representations of many noncompact groups ${ }^{15}$. Work on the geometric structure of the Dirac bracket in classical mechanics was an indication of Sudarshan's range of interests ${ }^{17}$.

\section{Texas before George}

During the early 1950s, the United States was the dominant world power and was regarded as the security needed to stem the threat of a growth of communism. The successful launch of Sputnik 1 by USSR on 4 October 1957 , and the failure of the US to counter with a successful launch raised serious concerns in the western countries but particularly in the US. This initiated a significant growth in the support of science research and education in the US. Prior to this, strong science, particularly basic university-based physics research, was concentrated on the east and west coasts and the Chicago area. The National Science Foundation initiated a major grant programme to build strong university research programmes, called Regional Development Grants, and Texas was a successful grantee. These grants were unusual in that they included capital expenditure for such items as buildings. The grant paid for two-thirds of the current physics building (note 3 ). It also included support for a new category of transitional faculty titled Faculty Associates who, after two years of research and some teaching, would become Assistant Professors on a tenuretrack line.

Prior to the award of the Regional Development Grant, the university and the Physics Department (note 4) were not ranked by any ranking organizations. In fact, the University had only two ranked programmes - linguistics and botany. As a part of the negotiations for the grant, the University committed to forming a separate Astronomy Department and expanded the size of the Physics Department. The Physics Department approximately doubled in size to over 45 faculty and, with the maturity of the Faculty Associates to Assistant Professors, to over 50 faculty-equivalent positions.

Another feature of the operations at UT at Austin at that time was a special funding source titled 'Available Fund'. This was the income from the sale of oil from University land in west Texas and was allocated to support academic excellence. This was accomplished through the establishment of research units called Centers. As a part of the negotiations for the Regional Development Grant, there was a commitment to add several 
Centers in the sciences. In particular, there were Centers for Particle Theory, Relativity, Experimental Plasma Physics, and Statistical Mechanics.

\section{George at Texas}

George Sudarshan joined the faculty of UT at Austin as a Professor of Physics and Co-Director (note 5) of the Center for Particle Theory. The Center was a budgeted line item which at the time of his joining had three senior faculty and several Faculty Associates. George brought his group from Syracuse along with funding from the AEC.

\section{George's Science}

George completed his work on underlying structure of classical mechanics and articulated the results of these studies in a book written with Mukunda ${ }^{7}$. He also began extending earlier work on indefinite metric field theories ${ }^{18}$ and applying the work to realistic field theory models ${ }^{19}$. Using results of his earlier work on the Poincaré group working with Mukunda, he developed a working scheme for constrained world lines in relativistic mechanics ${ }^{20}$.

His deep understanding of the Poincaré group also allowed him to do the most complete study on the possibility of signals or particles travelling at speeds higher than the speed of light. There are subtle questions related to the structure of a causal space-time and the quantum basis for our understanding of light. He extended his earlier studies ${ }^{21}$ to ultimately promote the most comprehensive understanding of these objects ${ }^{22}$.

One of the primary signatures of George's work has always been the application of new ideas used in particle theory and applying them to classical problems in physics $^{23}$ or putting sophisticated mathematics to use in particle theory ${ }^{24}$. In addition, he would solve classical mathematical problems or difficult philosophical ones with his powerful physical intuition ${ }^{25}$.

George had an abiding interest in the relation of particle spin and particle interchange symmetry in multi-particle systems and this continued at Texas. Bose had proposed Bose statistics for photons; Fermi and Dirac had shown that the Pauli exclusion principle could be translated into the statistics obeyed by spin $1 / 2$ particles. The history of ideas up to and including Pauli's work is systematically presented in a monograph 'Pauli and the Spin-Statistics Theorem' by Duck and Sudar$\operatorname{shan}^{26}$.

That the symmetry of the many-particle wave functions could be Bose, Fermi, or more general statistics was shown to depend on the connectivity of the configuration space. Sudarshan with Tom Imbo studied this question in a series of papers but these considerations did not yield the connection between spin and statistics ${ }^{25}$.
For quantum field theory, Pauli's proof depended on invoking relativistic invariance; but the major impact of particle statistics is in the non-relativistic domain such as atomic structure, nuclear structure, theory of metals, theory of superconductivity and the theory of phonons. Sudarshan showed that this connection can be obtained from the symmetry of scalars bilinear in a field. He also showed that this product is symmetric for integer spin and antisymmetric for half-integer spins. These results were presented in his paper in the Proceedings of the Indian Academy of Sciences and subsequent publications, including the monograph by Duck and Sudarshan, and in a paper with A. Shaji ${ }^{26}$.

Being the original discoverer of the Optical Equivalence Theorem ${ }^{9}$, Sudarshan was the best person to extend the understanding of how the light of our perception emerges from the photons of Planck. With many collaborators, he developed a comprehensive explanation of partial coherence, radiative transfer and nonlinear coherent states $^{27}$. His work in this area was monumental but again there was inadequate recognition (note 6).

The saying that a watched pot never boils is more than an admonition in quantum system. Careful analysis of the decaying process in quantum mechanics revealed three timescales for decay; the very short or Zeno, middle range or exponential and the very long or Khalfin. Zeno timescale had never been predicted or observed ${ }^{27}$. Systematic checking on an unstable atomic system verified the effect ${ }^{28}$.

The Zeno effect on decay lifetimes is part of the more general problem of quantum measurement. George's work on these problems goes back to his work at TIFR on the interpretation of nuclear tracks in photo-sensitive emulsions ${ }^{1}$ and threads through the Rochester and Syracuse years $^{21}$ but reaches a peak in Texas $^{3}$ when it was identified as a general problem of our understanding of the quantum measurement.

The problem has also taken on significance because of the growing importance of quantum information processes. Like the chemist with his pressure bath of the atmosphere, the modern computer with qubit elements will always have to operate in a background of classical objects $^{29}$. As always seems to be the case with George, he was way ahead of his times, or at least abreast of the most subtle issues.

\section{George's honours}

George's scientific contributions were recognized by many institutions - of the order of seven Honoris Causa $\mathrm{Ph}$ Ds from leading universities around the world. He also accumulated more than ten special honours from scientific institutions. His two most cherished of these awards are the Padma Bhushan (Order of the Lotus) presented by the President of India in 1976 and the Dirac Medal presented by the Institute of Theoretical Physics in Trieste, 
Italy, in 2010. The latter was shared with Nicola Cabibbo for their work on weak interactions. The Dirac Medal had special significance for two reasons. He held Dirac in his highest esteem of modern theoretical physicists. There is also an interesting twist to the Dirac Prize; it is considered by most physicists as the prize for people who should have gotten the Nobel Prize but were skipped over. It seems that George should have been the first person to receive two Dirac Medals.

\section{George's students and postdocs}

Besides the production of new knowledge, one of the main roles for an academic scientist is the development of a cadre of effective young scientists to carry the torch of scientific inquiry forward. George is likely to be among the most successful of the academic theoretical physicists in his generation in this process. The number of young postdocs and students he has worked with comes to more than 70 and more than $80 \%$ of them are serving as faculty at major universities or research laboratories in the US and abroad.

\section{George's publications}

During his long successful career in research, George produced an immense body of published results; he has more than 450 formally published papers. The references given below are for the use of readers interested in more detailed information about the issues discussed in the section 'George's Science'.

Another striking feature of his publications is the number of co-authors on his papers. Once out of Rochester, the pattern was George and a small group of younger physicists, postdocs and students. This is especially apparent even in the selected list of publications as given below but especially so if the conference and review presentations and books are removed from the list.

George also wrote seven textbooks ${ }^{30}$; listed as texts, these are more like monographs. They carry all the usual approach to subject matter suitable in an undergraduate or graduate classroom, but there is also a coherent exposition of material that is usually found in a research paper.

\section{Notes}

1. Older textbooks mention that there are four fundamental forces: the electromagnetic characterized by light, weak characterized by natural radioactivity, gravity, and strong characterized by nuclear stability. Now there are only three: the standard model combining the first two, gravity, and the strong force now identified as quantum chromodynamics.

2. Now called the Department of Energy.

3. The two-thirds was the result of the fact that the grant could not support the construction of classrooms, only research supporting facilities, including faculty offices.

4. There was not an Astronomy Department. Two members of the Physics Department taught the Astronomy classes. This was a particular anomaly since the University owned an observatory in the Davis Mountains. It was managed and operated by the University of Chicago.

5. Yuval Neéman was the other Co-Director. He came to Texas the year before Sudarshan. He was only half time at Texas and his other half was at Tel Aviv University in Israel.

6. Roy Glauber of Harvard University was awarded a Nobel Prize in Physics in 2005 for his work in this field.

1. An improved method for the determination of the mass of particles from scattering versus range and its application to the mass of $\mathrm{K}$ mesons; with Biswas, S. and Peters, B., Proc. Indian Acad. Sci., 1953, 38, 418 and Nuovo Cimento, Suppl., 1954, 12, 369 with Biswas, S., Peters, B. and Swamy, M. S. and Proc. Indian Acad. Sci., 1955, 41, with Daniel, R. R. and Peters, B.

2. Mass differences of $\Sigma^{ \pm}$and their anomalous magnetic moment. Phys. Rev., 1956, 104, 267 with Marshak, R. E. and Consequences of charge independence for magnetic moments and masses of the $\Sigma$ hyperons, Phys. Rev., 1957, 106, 599 with Okubo, S. and Marshak, R. E. and Interaction current in strangeness violating decays. Phys. Rev., 1958, 112, 665 with Okubo, S., Marshak, R. E., Teutsch, W. B. and Weinberg, S., reprinted in The Development of the Theory of Weak Interactions (ed. Kabir, P. K.), Gordon and Breach, New York, USA, 1964.

3. Irreversibility and dynamical maps of statistical operators; with Gorini, V., Lecture Notes in Physics, 260, 29; Springer Verlag, Berlin, 1974; Interaction between classical and quantum systems, a new approach to quantum measurements I with Sherry, T. N., Phys. Rev. D, 1978, 18, 4580; Interaction between classical and quantum systems and the measurement of quantum observables. Pramana, 1976, 6, 117; Interaction between classical and quantum systems, a new approach to quantum measurements II: theoretical considerations with Sherry, T. N., Phys. Rev. D, 1979, 20, 857; Interaction between classical and quantum systems, a new approach to quantum measurements III: illustration with Gautum, S. R. and Sherry, T. N., Phys. Rev. D, 1979, 20, 3081; Quantum dynamical semigroups and complete positivity. An application to isotropic spin relaxation. In IX International Colloquium on Group Theoretical Methods in Physics, Cocoyoc, Mexico, June 1980. In Proceedings Lecture Notes in Physics, vol. 135, Springer Verlag, Berlin; with V. Gorini and Verri.

4. The nature of the four-Fermion interaction. In Proceedings of the Conference on Mesons and Newly Discovered Particles, PaduaVenice, September 1957 and Chirality invariance and the universal Fermi interactions. Phys. Rev., 1958, 109, 1860, both with Marshak, R. E.

5. Inconsistency of the local field theory of charged spin $3 / 2$ particles. Ann. Phys., 1961, 13, 126 with Johnson, K.

6. Structure of the vertex function. Phys. Rev., 1959, 115, 731; structure of the forward scattering amplitude. Phys. Rev., 1960, 117, 266; and integral representation of two-point functions. Phys. Rev., 1960, 117, 272; with Deser, S. and Gilbert, W.

7. Classical Mechanics: A Modern Perspective, John Wiley and Sons, New York, with Mukunda, N., 1974.

8. Lie group dynamical formalism and the relationship between quantum mechanics and classical mechanics. Rev. Mod. Phys., 1961, 33, 515 with Jordan, T. F.

9. Equivalence of semiclassical and quantum mechanical descriptions of statistical light beams. Phys. Rev. Lett. D, 1963, 1, 277.

10. Fundamentals of Quantum Optics, W. A. Benjamin, New York, USA with Klauder, J., 1968.

11. Quantum mechanical systems with an indefinite metric I. Phys. Rev., 1961, 123, 2183 and Quantum mechanical systems with an indefinite metric II. Phys. Rev. 1961, 123, 2193; the latter with. Schnitzer, H. J.

12. 'Meta' relativity; with Bilaniuk, O. M. P. and Deshpande, V. K., Am. J. Phys., 1962, 30, 718-723. 
13. Introduction to Elementary Particle Physics, Interscience Publishers, New York, USA with Marshak, R. E., 1961.

14. Finite quantum electrodynamics: a field theory using an indefinite metric. Phys. Rev., 1965, 137, 1085 with Arons, M. E. and. Han, M. Y.

15. Application of noninvariance groups to Meson-Baryon scattering. Phys. Rev. Lett., 1966, 16, 825; Noninvariance groups from intermediate coupling models. Phys. Lett., 1966, 21, 106 and Noninvariance groups in particle physics. Phys. Rev., 1967, 162; all with Kuriyan, J. G.

16. Theory of photoelectric detection of light fluctuations. Proc. Phys., 1964, 84, 435 with Mandel, L. and Wolf, E.

17. Structure of the Dirac bracket in classical mechanics. J. Math. Phys., 1968, 9, 411 with Mukunda, N.

18. Analyticity, covariance and unitarity in indefinite metric quantum field theories. Phys. Rev. D, 1971, 4, 2242 with Moore, R. M., Gleeson, A. M. and Rechenberg, H.

19. Quantum field theories with shadow states I. Soluble models. Phys. Rev. D, 1972, 6, 3658 and Quantum field theories with shadow states II. Low energy Pion-Nucleon scattering. Phys. Rev. D, 1972, 6, 3678 with Nelson, C. A.

20. Constraint dynamics of particle world lines, with Mukunda, N and Goldberg, J., Phys. Rev. D, 1981, 23, 2218 and Form of relativistic dynamics with world lines with Mukunda, N., Phys. Rev. D, 1981, 23, 2210.

21. Stochastic dynamics of quantum-mechanical systems with Mathews, P. M. and Rau, J., Phys. Rev., 1961, 121, 920; Dynamical mappings of density operators in Quantum-Mechanics II. Time dependent mappings; with Jordan, T. F. and Pinsky, M. A., J. Math. Phys., 1962, 3, 848 and Study of spurious scattering in nuclear emulsions and the effect of higher order differences in scattering measurements with Lavakare, P. J., Nuovo. Cimento, Suppl., $1962,20,251$

22. Lorentz invariance, local field theory, and faster than light particles; with Arons, M. E., Phys. Rev., 1968, 173, 1622 and Quantum field theory of interacting tachyons with Dhar, J., Phys. Rev., 1968, 174, 1808, Causality and spacelike signals with Bilaniuk, O. M. P., Nature, 1969, 223, 386; Particles beyond the light barrier with Bilaniuk, O. M. P., Phys. Today, 1969, 22, 43. Tachyon cloud of a particle. Phys. Rev. D, 1, 1970, 2428. The theory of particles traveling faster than light I, Symposia on theoretical physics and mathematics (ed. Ramakrishnan, A.), Plenum Press, New York, 1970 and Tachyons and cosmology with Narlikar, J. V., Month. Not. R. Astron. Soc., 1976, 175, 105.

23. Nonabelian monopoles break color I: classical mechanics; with Balachandran, A. P., Marmo, G., Mukunda, N., Nilsson, J. and Zaccaria, F., Phys. Rev. D, 1984, 29, 2919

24. Quantum measurement and dynamical maps. From SU(3) to gravity. In Festschrift in Honor of Yuval Ne'eman (eds Gotsman, E. and Tauber, G.), Cambridge University Press, Cambridge, 1986, p. 433; Quantum dynamics, metastable states and contractive semigroups. Phys. Rev. A, 1992, 46, 37; Unstable systems in generalized quantum theory with Charles B. Chiu and Bhamathi, G., In Advances in Chemical Physics XCIX, John Wiley, 1997, p. 121.

25. Topological and algebraic aspects of quantization: symmetries and statistics; with Tom Imbo and Chandni Imbo. Ann. Inst. Henri
Poincaré, 1988, 49, 387 and Inequivalent quantization in multiplyconnected spaces. Il; with Horvathy, P. A. and Morandi, G., Nuovo Climate, 1989, 20, 201.

26. The fundamental theorem on the relation between spin and statistics. Proc. Indian Acad. Sci., 1968, LXVII, 284 and A world of Bose particles. Science Today, January 1974, Am. J. Phys., 1975, 43(1), 69 and Pauli and the Spin-Statistics Theorem, World Scientific, Singapore, 1998 and Toward an understanding of the spinstatistics theorem and Am. J. Phys., 1998, 66(4), 28, both with Ian Duck and non-relativistic proof of spin-statistics theorem with Shaji, A., 2003; http://arxiv.org/pdf/quant-ph/0306033.

27. Khalfin, L. A., Zh. Eksp. Teor. Fiz., 1957, 33, 1371; Sov. Phys JETP, 1958, 6, 1053. The Zeno's paradox in quantum theory with Misra, B., J. Math Phys., 1977, 18(4), 756; Time evolution of unstable quantum states and a resolution of Zeno's paradox; with Chiu, C. B. and Misra, B., Phys. Rev. D, 1977, 16, 520; The time scale for quantum Zeno paradox and proton decay with Misra, B. and Chiu, C. B., Phys. Lett. B, 1982, 117, 34; Decay and evolution of the neutral Kaon with Chiu, C. B., Phys. Rev. D, 1990, 42, 3712; Unstable systems in generalized quantum theory with Charles B. Chiu and Bhamathi, G., Advances in chemical physics XCIX, John Wiley, 1997, pp. 121-210; Quantum Zeno dynamics; with Facchi, P., Gorini, V., Marmo, G., Pascazio, S., Phys. Lett. A, 2000, 275, 12 and Zeno dynamics with constraints with Facchi, P., Marmo, G., Pascazio, S. and Scardicchio, A., J. Opt. B, 2004, 6, S492.

28. Quantum Zeno effect, Wayne, M., Itano, D. J., Heinzen, J. J. Bollinger and Wineland, D. J., Phys. Rev. A, 1990, 41, 2295 and Observation of the quantum Zeno and anti-Zeno effects in an unstable system. Fischer, M. C., Gutierrez-Medina, B. and Raizen, M. G., Phys. Rev. Lett., 2001, 87, 040402

29. Mapping the Schrodinger picture of open quantum dynamics with Jordan, T. and Anil Shaji; http://arxiv.org/pdf/quant-ph/0505123, 2004, Dynamics of initially entangled open systems with Shaji, A. and Jordan, T., Phys. Rev. A, 2004, 70, 052110 and on the meaning and interpretation of tomography in abstract Hilbert Spaces with Manko, V. I., Marmo, G. and Zaccaria, F., Rep. Math. Phys., $2005, \mathbf{5 5}, 405$.

30. Introduction to Elementary Particle Physics, Interscience Publishers, New York, USA, 1961; with Marshak, R. E., Fundamentals of Quantum Optics, W. A. Benjamin, New York, USA, 1968 with Klauder, J.; Classical Mechanics: A Modern Perspective, John Wiley, New York, USA, 1974; with Mukunda, N.; Pauli and the spinstatistics theorem, World Scientific, Singapore, 1998 with Ian Duck; 100 Years of Planck's Quantum, World Scientific, Singapore, 2000; with Ian Duck; From Classical to Quantum Mechanics: An Introduction to the Formalism, Foundations and Applications, Cambridge University Press, Cambridge, UK, 2010 with Giampiero Esposito, Giuseppe Marmoand; Advanced Concepts in Quantum Mechanics, Cambridge University Press, Cambridge, UK, 2014 with Giampiero Esposito, Giuseppe Marmo and Gennaro Miele.

31. Proceedings of the 1974 Conference on Neutrinos-1974, R. P. Feynman clarifying priority on $\mathrm{V}-\mathrm{A}$ theory, p. 300.

doi: $10.18520 / \mathrm{cs} / \mathrm{v} 116 / \mathrm{i} 2 / 211-215$ 